J. Clin. Chem. Clin. Biochem.

Vol. 15, 1977, pp. 333-337

\title{
Urinproteinanalysen mit der Diskelektrophorese: Ein Verfahren zur Differentialdiagnose von Nieren- erkrankungen
}

\section{Von H. Rautenstrauch}

Abteilung für Nephrologie und Rheumatologie des Zentrums für Innere Medizin (Chefarzt: Prof. Dr. G. Seybold) am Robert-Bosch-Krankenhaus, Stuttgart

(Eingegangen am 28. Juni/17. Dezember 1976)

Zusammenfassung: Nach einleitenden Bemerkungen über die Rolle der Niere im Proteinstoffwechsel werden die Methode der Diskelektrophorese auf Polyacrylamid unter Verwendung von Detergentien und ihre Ergebnisse beschrieben. Die Auftrennung der Urinproteine nach dem Molekulargewicht erlaubt eine Differenzierung von Nierenerkrankungen in solche glomerulärer und tubulärer Genese, außerdem sind Proteinurien prärenalen und postrenalen Ursprungs ab. grenzbar. Der beträchtliche Zeitaufwand verhindert zunächst einen Einsatz im Routinebetrieb.

\section{Analysis of urinary proteins by disc electrophoresis: $A$ method for the differential diagnosis of kidney diseases}

Summary: After introductory observations on the role of the kidney in protein metabolism, the methodology and results of disc electrophoresis on polyacrylamide, using detergents, are discussed. Separation of urinary proteins according to molecular weight permits the differentiation of kidney diseases into those of the glomerulus and of the tubules. Proteinurias of prerenal and postrenal origin are also differentiated. Owing to the considerable time involved, this methodology cannot at present be introduced for routine purposes.

\section{Einleitung}

Die Rolle der Niere im Stoffwechsel der Plasmaproteine ist in den letzten Jahren zum Teil aufgeklärt worden (1), in vieler Hinsicht aber auch noch Gegenstand intensiver Forschung (2). Zum Verständnis der in dieser Arbeit dargestellten Methode seien die gesicherten Erkènntnisse kurz beschrieben.

Proteine niedrigen Molekulargewichtes $(<50000)$ pas: sieren das glomeruläre Filter und werden durch die Tubuluszellen aufgenommen und zu Aminosäuren abgebaut. Für diese Klasse von Proteinen ist die Niere ein primäres Organ des Katąbolismus. Bei tubulären Erkrankungen sind Reabsorption und Abbau dieser Proteine niederen Molekulargewichts gestört, und sie werden im Urin nachweisbar. Proteine mittleren und hohen Molekulargewichtes $(>60000)$ werden glomerulär retiniert und passieren die Nieren nur bei Alteration des glomerulären Filters. Der Nachweis dieser Proteine im Urin gibt einen Hinweis für eine glomeruläre Schädigung.

Die Diskelektrophorese auf Polyacrylamid unter Detergentien (Natrium-Dodecylsulfat) ermöglicht eine Auf- trennung der Urinproteine nach dem Molekulargewicht und erlaubt dadurch eine Differenzierung von Nierenerkrankungen in solche glomerulärer und tubulärer Genese. Außerdem sind Proteinurien prärenalen (BenceJones-Proteinurie) und postrenalen (Immunglobulinsekretion im Tubulus) Ursprungs abgrenzbar (3-6).

Gegenüber anderen Methoden zur Differenzierung der Proteinurien (Gelfiltration, Ultrazentrifugation, Immundiffusion) besitzt die Diskelektrophorese die Vorteile geringen Zeitaufwandes und größerer Präzision $(1,3)$. Die weit verbreitete Acetatfolien- bzw. Papierelektrophorese ist für die gegebene Fragestellung ungeeignet, da Fraktionen, die unterhalb des Molekulargwichts des Albumins liegen, nicht erfaßt werden. Die neuerdings angegebene Mikroelektrophorese in kontinuierlichen Polyacrylamid-Gradientengelen (7) kann ohne die zeitraubende Urinkonzentration erfolgen und erfordert nur eine kurze Trennzeit, ein Vorteil, wenn die methodisch erforderliche „Urinkonzentration von 0,1 bis $1,0 \mathrm{~g} / 1$ Protein" (7) vorliegt oder sogar überschritten ist. Die Aussagefähigkeit der Methode bei niedrigeren Urinproteinkonzentrationen ist noch nicht eindeutig belegt. 


\section{Methodik}

$50 \mathrm{ml}$ Morgenurin werden mit Labstix auf Blut, pH, Protein geprüft, zentrifugiert und dann entsprechend ihrem ProteinGehalt 100- bzw. 200-fach konzentriert. Die Konzentration erfolgt mit Kollodiumhülsen (Sartorius-Membranfilter SM 13200 ), einer Haltevorrichtung (SM 263 14) und einer Vakuumpumpe (Drehschieberpumpe v. Leybold-Heraeus). Die konzentrierten Urine werden bei $-20^{\circ} \mathrm{C}$ aufbewahrt.

Die Diskelektrophorese wurde mit der Flachgel-ElektrophoreseApparatur von Desaga durchgefürr.

\section{Reagenzien}

Elektrophorese-Puffer

$0,1 \mathrm{~mol} / 1 \mathrm{sek}$. Natriumphosphat, $17,3 \mathrm{mmol} / 1$ Natriumdodecylsulfat mit $1 \mathrm{~mol} / \mathrm{l} \mathrm{HCl}$ auf $\mathrm{pH}$ 7,40 einstellen (Merck).

\section{$7.5 \mathrm{~g} / \mathrm{l}$ Polyacrylamid Gel}

$4050 \mathrm{mg}$ Acrylamid und $105 \mathrm{mg}$ Bisacrylamid werden in $50 \mathrm{ml}$

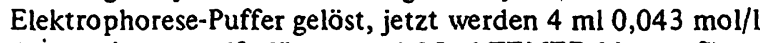
Ammoniumpersulfatlösung und $25 \mu$ l TEMED hinzugefügt (alle Substanzen von Fa. Serva).

\section{Arbeitswe ise}

Die Lösung für das Gel wird, wie oben beschrieben, zubereitet, gut gemischt und in den Arbeitsblock (Desaga) gefüllt, mit dest. Wasser überschichtet, dann wird der Teflon-Kamm für $10 \mathrm{Gel}-$ taschen hineingesteckt. Die Polymerisationsdauer beträgt 30 min. Während der Polymerisation wird der Block gekühlt und nach Beendigung das überschichtete Wasser wieder vorsichtig abgesaugt. Der Arbeitsblock wird in die Trennkammer eingebaut und der Elektrophorese-Puffer eingefüllt.

\section{Probenvorbereitung}

$50 \mu$ Elektrophorese-Puffer, $20 \mu l$ konzentrierten Urin, $5 \mu \mathrm{l}$ Glycerin und $5 \mu \mathrm{l} 0,5 \mathrm{~g} / \mathrm{l}$ Bromphenolblaulösung gut mischen und mit einer Hamilton-Spritze $20-40 \mu l$ pro Geltasche auftragen.

Die Trennung erfolgt bei 60-70 mA (Netzgerät v. Desaga), Dauer 5-6 h. Die Färbung wird mit $8 \mathrm{~g} / \mathrm{l}$ Amidoschwarz in $1,16 \mathrm{~mol} / 1$ Essigsäure durchgeführt, Dauer 2 Stunden.

Die Entfärbung in $1,16 \mathrm{~mol} / 1$ Essigsäure erfolgt elektrophoretisch, Entfärbungsdauer etwa 10 Stunden. Die Auswertung erfolgt mit dem Vitatrondensitometer, der Zeitbedarf beträgt bei 10 Proben etwa 1 Stunde.

\section{Ergebnisse}

Im folgenden sind einige typische Befunde, die mit der beschriebenen Methode erhoben werden konnten, zusammengestellt.

Abbildung 1 ist ein Originalstreifen einer PolyacrylamidNatriumdodecylsulfat-Elektrophorese mit 10 aufgetragenen Urinproben.

Albumin (Molekulargewicht 65000 Dalton) stellt fast immer die Hauptkomponente, bei Untersuchung eines Normalurins die einzige Komponente dar. Banden mit einem Molekulargewicht über 65000 liegen in unserem Beispiel kathodenwärts von Albumin, die mit einem Molekulargewicht unter 65000 anodenwärts. Eine sichere Charakterisierung der einzelnen Banden ist zu

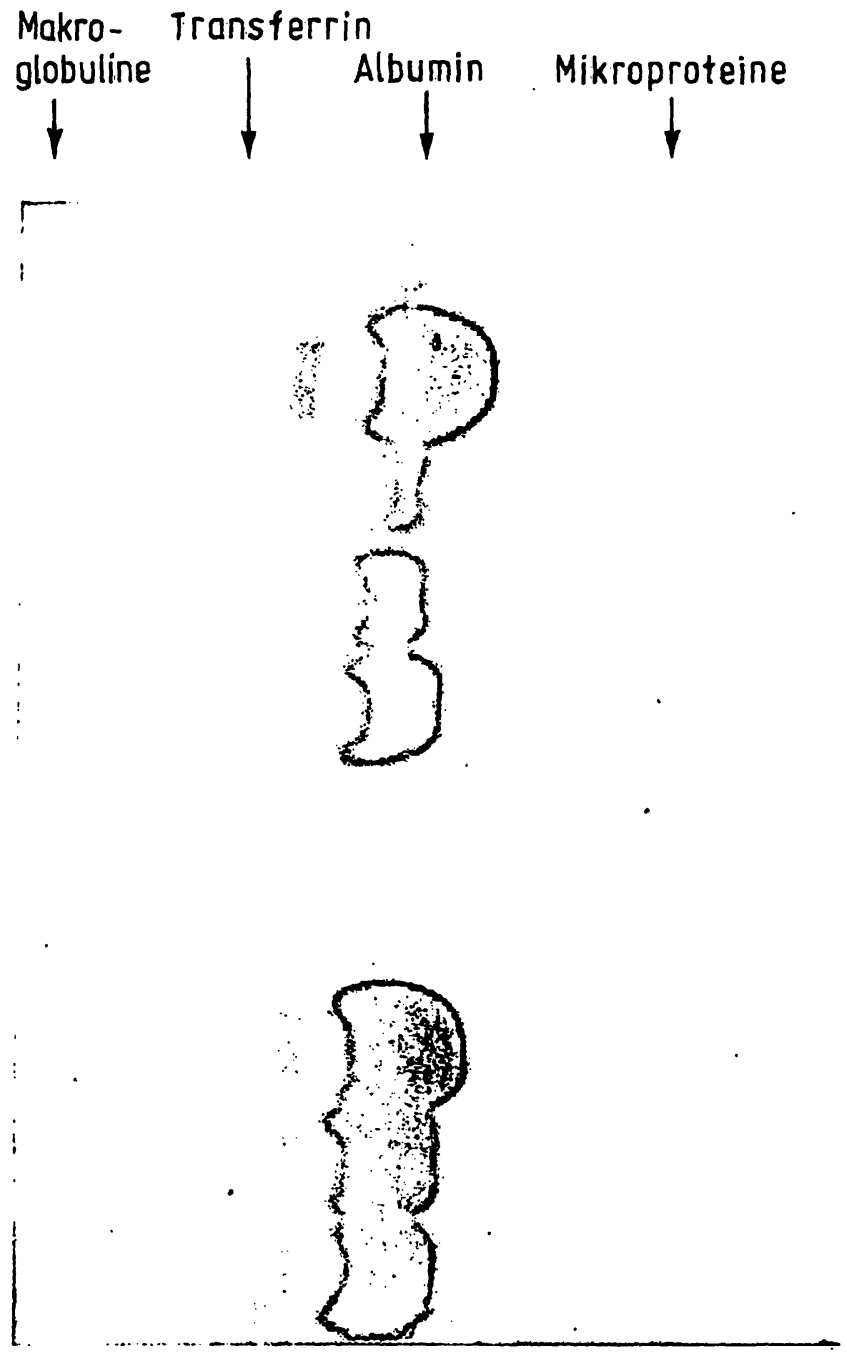

Abb. 1. Mit Polyacrylamidgel-Natriumdodecylsulfat-Elektrophorese getrennte Fraktionen nạch Anfärbung.

erreichen, wenn man sie mit der Mobilität von Proteinen bekannten Molekulargewichtes vergleicht oder sie immunchemisch untersucht. In der Praxis gestatten visuelle Beurteilung und Erfahrung eine Differenzìerung der getrennten Fraktionen, vor allem unter Benutzung der in der Folge gezeigten densitometrischen Kurven.

Abbildung 2a zeigt den Befund einer tubulären Proteinurie bei bakterieller interstitieller Nephritis.

Links sieht man einen kleinen Transferrinpeak, rechts neben dem doppelgipfligen Albumin finden sich vier verschiedene Fraktionen niedermolekularer Proteine. Generell sind fünf Mikroproteinfraktionen möglich, die auch mit $\overline{\mathrm{T}}_{1}-\mathrm{T}_{5}$ bezeichnet werden $(3,8)$. Es handelt sich dabei um $\alpha_{1}$-Antitrypsin (MG 54 000), freie Leichtketten der Immunglobuline in di- (MG 44 000) und monomerer Form (MG 22 000), Retinol-bindendesProtein (MG 21 000) und $\beta$-2-Mikroglobulin (MG 12 000).

Proteine, deren Molekulargewicht unter 10000 liegen, werden mit der Diskelektrophorese aus methodischen Gründen nicht erfaßt. 
Abbildung $2 \mathrm{~b}$ gibt die Veränderungen einer selektiven glomerulären Proteinurie bei „minimal change nephritis" wieder.

Neben dem stark ausgeprägten Albuminpeak sieht man einen kleineren Transferrinpeak.

Abbildung $2 c$ zeigt eine unselektive glomeruläre Proteinurie bei immunhistologisch gesicherter perimembranöser Glomerulonephritis. Neben Albumin und Transferrin sieht man zusätzlich eine IgG-Bande, möglicherweise als Ausdruck einer Baselmembranschädigung. Bei dem Beispiel der folgenden Abbildung 2d handelt es sich ebenfalls um eine unselektive glomeruläre Proteinurie bei diabetischer Glomerulosklerose und Arterio-Arteriolosklerose der Nieren. Im Unterschied zum vorhergehenden Diskelektrophoresebild wird neben Albumin, Transferrin und IgG auch IgM ausgeschieden. Man sieht also, daß bei unselektiver glomerulärer Proteinurie durchaus Variationen möglich sind. Charakteristisch ist jedoch, daß die Molekulargewichte der ausgeschiedenen Proteine zwischen 65000 und 1000000 liegen. Kennzeichen der selektiven Proteinurie ist hingegen, daß nur Proteine mit einem Molekulargewicht zwischen 65000 und 100000 in den Urin übertreten.

Die Proteinbanden der glomerulären Proteinurie wurden entsprechend der tubulären auch mit $G_{1}, G_{2}$ (Transferrin), $G_{3}, G_{4}\left(7^{S}-I g G\right), G_{5}$ und $G_{6}\left(\alpha_{2}\right.$-Makroglobulin, IgM) bezeichnet.

Tubuläre und glomeruläre Proteinurien kommen häufig zusammen vor (s. Abb. 2e). Ein Maß zur Differenzierung solcher gemischter Proteinurien ist die GlomeruloTubuläre Protein Ratio (GTRR = Menge der Proteine MG $>90$ 000/Menge der Proteine MG <65 000), die bei überwiegend tubulären Formen unter 1 legt (3).

In der Abbildung $2 \mathrm{f}$ sind eine prärenale (Bence-Jones) bei gleichzeitiger postrenaler (tubuläre IgG-Sekretion) Proteinurie bei Plasmocytom wiedergeben. Neben der
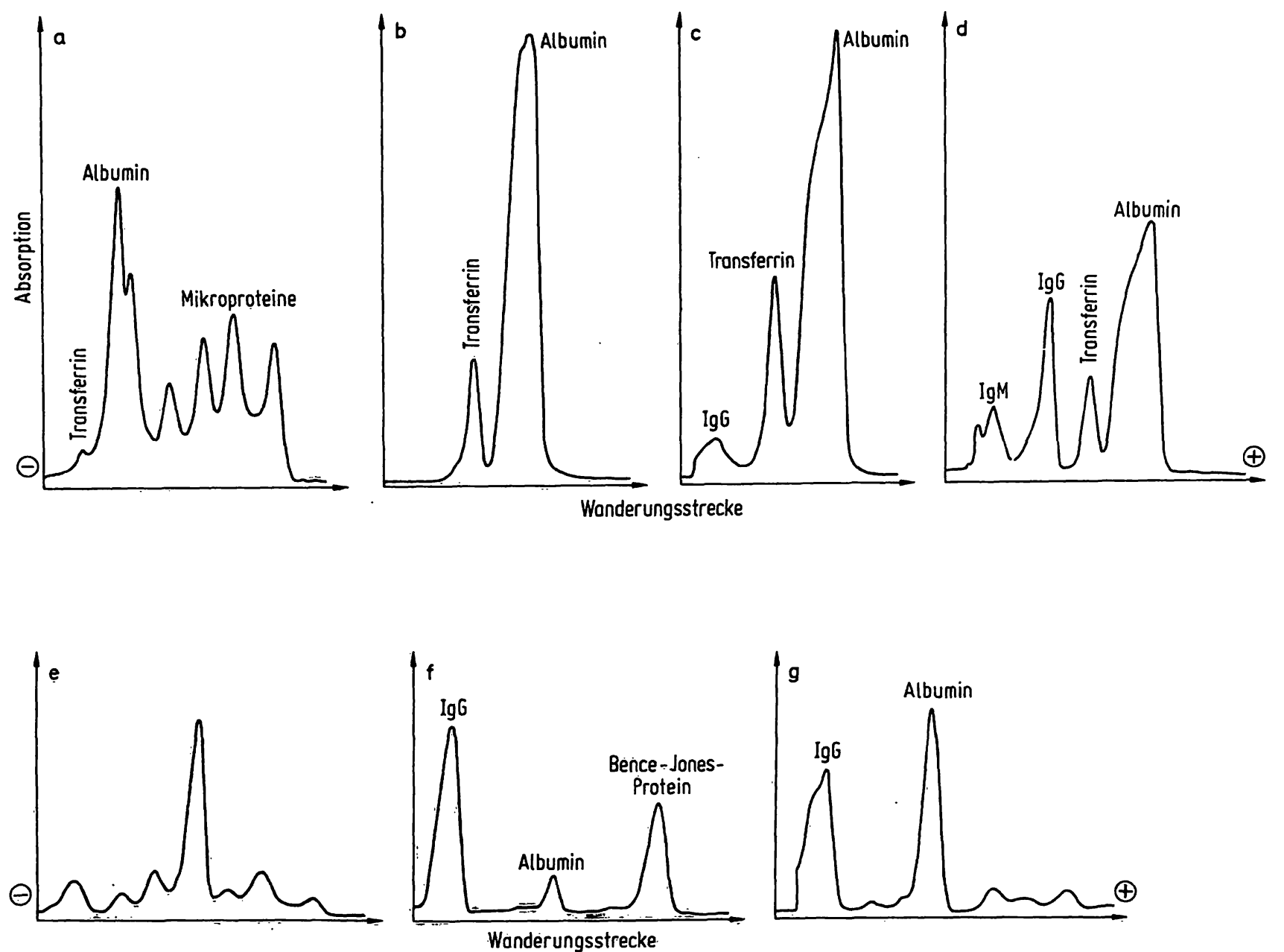

Abb. 2. Densitogramme von mit Polyạcrylamid-Natriưmdodeçylsulfat-Elektrophorese getrennten Harnproteinen.

a. Tubuläre Proteinurie bei bakterieller interstitieller Nephritis.

b. Selekt ive glomeruläre Proteinurie bei "minimal change nephritis“.

c. Unselèktive glomeruläre Proteinurie bei perimembranöser Glomerulonephritis.

d. Unselektive glomeruläre Proteinurie bei diabetischer Glomerulosklerose und Arterio-Arteriosklerose der Nieren.

e. Tubuläre und glomeruläre Proteinurien.

f. Prärenale (Bence-Jones) und gleichzeitig postrenale (tubuläre IgG-Sekretion) Proteinurie bei Plasmocytom.

g. Tubuläre Proteinurie mit begleitender tubulärer İmmunglobulinausscheidung. 
kleinen Albuminzacke in der Mitte sieht man anodenwärts das Bence-Jones-Protein und kathodenwärts das IgG. Die Monoklonalität wurde durch Immunelektrophorese gesichert.

Die isolierte Ausscheidung von Immunglobulinleichtketten in dimerer Form (Molekulargewicht 44000 ) unterscheidet die prärenale Proteinurie von der in $\mathrm{Ab}$ bildung 2 a dargestellten tubulären Proteinurie, bei der mehrere Proteine aus dem Ultrafiltrat nicht aufgenommen werden. Infolge der hohen renalen Clearance der BenceJones-Proteine entstehen hohe tubuläre Konzentrationen, die die vorhandene Resorptionskapazität überschreiten und den Nachweis im Urin ermöglichen. Das IgG wird im vorliegenden Beispiel tubulär sezerniert, da bei glomerulärer Schädigung zusätzlich Transferrin ausgeschieden werden müßte.

Postrenale Proteinurien kommen oft als begleitende Imimunglobulinausscheidung in Verbindung mit einer tubulären Proteinurie, z. B. im Rahmen einer Urosepsis, vor (Abb. 2g). Auch hier erlaubt die nur geringfügige Transferrinurie den Ausschluß einer glomerulär bedingten Form. teinurien bei interstitieller Nephritis, akutem Nierenversagen, Stoffwechselerkrankungen (Cystinose, Oxalose), degenerativen Tubulusschäden, nach Kadavernierentransplantation; seltener im Ablauf schwerer Allgemeinerkrankungen (z. B. Endocarditis lenta, Colitis ulcerosa, M. Crohn) und als Begleiterscheinung primärer glomerulärer Erkrankungen.

Bei Proteinurien glomerulären Ursprungs kann man formal zwischen einer selektiven und einer, häufiger zu beobachtenden, unselektiven Form unterscheiden (Abb. 2b-d). Diese Begriffe bezeichnen wahrscheinlich einen funktionellen Zustand. Eine sichere Zuordnung zu bestimmten histologischen Bildern einer Glomerulonephritis ist nach unseren Erfahrungen nicht möglich. Eine glomeruläre Proteinurie, ob selektiv oder unselektiv, zeigt jedoch immer eine glomeruläre Schädigung an und wurde bei den unterschiedlichen Formen der Glomerulonephritis, bei diabetischer Glomerulosklerose, Arterio-Arteriolosklerose, Amyloidose und, überraschenderweise, bei der orthostatischen Proteinurie gefunden $(3,6,8,11,12)$. Eine massive glomeruläre Proteinurie über $8 \mathrm{~g} / \mathrm{d}$ kann den Nachweis von Mikroproteinen unmöglich machen.

Die beschriebene Methode ist eine wertvolle Hilfe in der Differentialdiagnose von Nierenerkrankungen. Da die Untersuchungen beliebig oft wiederholt werden können, eignet șich das Verfahren besonders für Verlaufsbeobachtungen und für die Feststellung der Ausheilung einer renalen Schädigung. Aussagen können nicht nur bei quantitativ erhöhter Proteinausscheidung, sondern auch bei physiologischen Proteinurien gemacht werden. Dabei ist eine 200-fache Konzentrierung des Urins erforderlich.

Eine quantitative Beurteilung der getrennten Proteine ist zwar bei Vorhandensein eines leistungsfähigen Derisitometers mit bestimmten Einschränkungen möglich (2). Sie bringt aber in der klinischen Praxis gegenüber der quantitativen Aussage (sichere Differenzierung von glomerulären und tubulären Proteinurien sowie Abgrenzung prärenaler und postrenaler Proteinurien) keinen entscheidenden Vorteil. So wird z. B. die Mikroproteinclearance nicht nur vom Ausmaß des tubulären Schadens, sondern auch von der glomulären Filtration dieser Proteine mit einem Molekulargewicht unter 65000 beeinflußt. Während die 24-Stunden-Eiweißausscheidung bei chronisch-interstitieller Nephritis meist unter $2 \mathrm{~g}$ liegt, finden sich nach Nierentransplantation Werte bis zu $5 \mathrm{~g}$. In beiden Fällen sind neben Albumin Mikroproteinfraktionen nachweisbar, deren quantitative Erfassung keinen Informationsgewinn darstellen würde. Eine Kontrolle über die Zuverlässigkeit der gewonnenen Ergebnisse ist dadurch möglich, daß man entweder Proteine bekannten Molekulargewichtes oder bereits früher getrennte Urinproben von Patienten mit bekannter Grunderkrankung nochmals mitlaufen läßt. Bei mehrfacher Analyse eines
Aufgrund vergleichender histologischer und diskelektro'phoretischer Untersuchungen anderer Autoren (3, 6, 8, $11,12)$ und eigener Ergebnisse finden sich tubuläre Pro- 
Urins des gleichen Patienten konnte immer das gleiche „Eiweißbild" reproduiert werden.

Ein Nachteil für den Routinebetrieb ist der beträchtliche Zeitaufwand (einschließlich der Urinkonzentrierung für 10 Urinproben 2 Tage, bei etwa 4 Stunden reiner Arbeits- zeit), die Methode muß deshalb zunächst speziellen Fragestellungen vorbehalten bleiben.

\section{Danksagung}

Frl. U. Krüger danke ich für die technische Assistenz.

\section{Literatur}

1. Strober, W. \& Waldmann, Th. A. (1974), Nephron 13, 35-66.

2. Deen, W. M., Chang, R. L. S., Robertson, C. R., Benett, C. M., Glassok, R. J. \& Brenner, B. M. (1975), VI th International Congress of Nephrology, Florenz 8.-12, 1975. Abstracts of Symposia.

3. Boesken, W., Kopf, K. \& Schollmeyer, P. (1973), Clin. Nephrol. 1, 311-318.

4. Pesce, A. J., Boreisa, I. \& Pollack, V. J. (1972), Clin. Chim. Acta 40, 27-34.

5. Pesce, A. J. (1974), Nephron 13, 93-104.

6. Virella, G., Pires, M. T. \& Maraves Coelho, J. (1974), Clin. Chim. Acta 50, 63-75.
7. Reichel, W., Wolfrum, D. I., Klein, R. \& Scheler, F. (1976), Klin. Wochenschr. 54, 19-24.

8. Boesken, W. (1975), Klin. Wochenschr. 53, 473-479.

9. Knox, G. F. \& Schneider, E. G. (1974), Nephron 13, 67-81.

10. Batsford, S., Bohnart, C., Boesken, W. \& Kluthe, R. (1975), Abstract, Kidney Int. 8, 405.

11. Höfer, W., Missgeld, W. \& Baethker, R. (1974), Protides Biol. Fluids, Proc. Colloq. 21, 459-462.

12. Wibell, L. \& Evrin, P. E. (1974), Protides Biological Fluids, Proc. Colloq. 21, 519-523. 
